\title{
COVID19 Infection in Health Care Professionals: Risks, Work- Safety and Psychological Issues
}

\author{
Yogesh G. Dabholkar ${ }^{1,3}$ (1) Bhagyashree Ajit Sagane ${ }^{1} \cdot$ Twinkle Y. Dabholkar $^{2}$. \\ Shivakumar Divity ${ }^{1}$
}

Received: 14 June 2020/ Accepted: 20 June 2020/Published online: 27 June 2020

(C) Association of Otolaryngologists of India 2020

\begin{abstract}
COVID-19 infection has spread widely over past 5 months to become a pandemic of global proportions affecting almost every country. While HCPs are expected to tackle this crisis by working in hospital and intensive care setting, there is real risk of them contracting infection and even dying. This article aims to report cases of healthcare personnel (HCPs) contracting COVID19 in various settings in a tertiary care hospital, a designated COVID centre, with view to disseminate information and review safety and psychological health issues of healthcare professionals. This study is a cross-sectional hospital-based survey from April 2020-June 2020. Data on demographics, workplace safety and psychological parameters from HCPs was collected by both interview and an online questionnaire form. A total of 40 healthcare workers were infected in the hospital in a period of 2 months since the first COVID case was admitted in the hospital. Almost 57.5\% reported positive on several psychological parameters like anxiety, fear, anger, irritability and insomnia. About $42.5 \%$ had no psychological counselling after testing positive. These cases illustrate work-place risks for healthcare workers of acquiring COVID19 and highlight the problems faced in terms of risks of transmission to patients and colleagues, isolation of contacts in departments leading to near-breakdown of services and psychological stress to healthcare workers. Healthcare workers being at frontline
\end{abstract}

Yogesh G. Dabholkar

ygsh@yahoo.com

1 Department of ENT, School of Medicine, D.Y. Patil University, Navi Mumbai, India

2 School of Physiotherapy, D.Y. Patil University, Nerul, Navi Mumbai, India

3 Navi Mumbai, India of exposure to corona patients are at increased risk of developing COVID19 infections. Healthcare workers are working under tremendous stress in this pandemic and it is necessary to combat fear with facts and work towards safe work atmosphere so that they can discharge their duties to best of their ability.

Keywords COVID19 · Work-safety · Psychological parameters · Hcps

\section{Introduction}

Coronavirus disease 2019 (COVID-19) caused by severe acute respiratory syndrome coronavirus 2 (SARS-CoV-2) is an ongoing pandemic of lower respiratory tract disease [1]. As such, rapid dissemination of information about origin as well as mode of transmission of this virus is central for disease control and implementing preventive measures and curative strategies [2]. Corona viruses exist in diameter range of $65-125 \mathrm{~nm}$ with single stranded RNA as nuclear material [3]. First reports of this disease date back to December 2019 from Wuhan province of China where it supposedly originated from an animal market and was named novel coronavirus pneumonia (NCP) on basis of sequence based analysis of isolates from the patients [4]. Increasing evidence documented human-to-human transmission [5, 6]. A quarantine period of 14 days was recommended for exposed contacts [6]. With the disease rapidly spreading worldwide, it was subsequently renamed as COVID-19 by World Health Organization, while the virus was named as SARS-CoV-2 by International Committee on Taxonomy of Viruses [7].

Spread outside China was initially to other countries in Asia, like Korea, Iran, then to EU, most notably Italy, 
Spain, the UK, the USA and finally to entire world with rapidly growing numbers everywhere with air travel. It was declared as global health emergency on January 30th 2020 by WHO $[5,8]$.

In India where numbers were initially low, it has grown steadily with cases doubling every 10-12 days although severe measures like lockdown were instituted to flatten the curve. While there has been regular tracking and reporting of cumulative cases and deaths across world, contribution to these numbers through work-place exposures has to be regularly analysed and published to develop strategies to prevent such spread. Clearly, workers involved in healthcare are at front line as regards risk of infection and death, like previous epidemics of severe acute respiratory syndrome (SARS) and middle east respiratory syndrome (MERS) [7, 8]. A physically and psychologically sound and well-equipped healthcare workforce is vital to a country's capability to manage COVID-19 pandemic. Lessons learnt from the pandemic response can help in launching innovative measures for the long-term.

This article aims to report cases of healthcare personnel (HCPs) contracting COVID19 in a tertiary care hospital, a designated COVID centre, with view to disseminate information and review safety and psychological health issues of healthcare professionals.

\section{Methods}

This study is a cross-sectional hospital-based survey from April 2020 to June 2020.

All HCPs who had been detected as COVID19 positive on a reverse-transcriptase-polymerase-chain-reaction (RTPCR) assay of clinical specimens obtained either from nasopharynx or oropharynx as per ICMR and WHO guidelines. Data on demographics of HCPs was collected by interviewing core team members of hospital Covid command team and also reviewing hospital information system. Data from HCPs was collected by both interview and an online questionnaire form. The questionnaire comprised of questions on demography, Covid19 symptoms, work-role, workplace-safety and psychological parameters. Those HCPs who failed to answer questionnaire were contacted on phone and interviewed after informed consent.

Descriptive statistics were used to summarise data as counts and percentages.

\section{Results}

A total of 40 healthcare workers were infected in the hospital in a period of 2 months since the first COVID case was admitted in the hospital. The hospital as soon as it was made a designated COVID centre, had established a central leadership command team, a dedicated COVID isolation ward, a dedicated COVID ICU, a Fever OPD, as per the directives of the Government. There were teams to overcome logistics and communication problems and provide counselling and support to all HCPs. All staff had access to adequate PPEs and regular training online and offline about infection control and PPE donning and doffing.

As soon as HCPs had unprotected contact with Covid positive patients or reported symptoms, hospital infection control team would ask them to undergo self-isolation and test them for Covid19. In the event of HCP testing positive, all contacts would be meticulously traced and tested for Covid19. HCPs would then be quarantined if asymptomatic or admitted in isolation units if symptomatic and monitored for temperature and pulse oxygen saturation.

Demographics of Covid19 positive HCPs are given in Tables 1 and 2.

Distribution of HCPs as per their role is shown in Table 3.

Of the five residents, three were from medicine department and one from otolaryngology and paediatrics each.

All HCPs who tested positive reported having access to adequate PPEs at work and adequate training. All received hydroxychloroquine (HCQ) prophylaxis, vitamin C supplements. Those who developed fever received anti-pyretic. Around $62.5 \%$ reported having either or all of symptoms of fever, headache and weakness. $37.5 \%$ were asymptomatic. Two HCPs (5\%) had become breathless requiring oxygen administration and of these one required admission in ICU. Both were given antivirals.

47.5\% HCPs felt that they had acquired infection at workplace with $15 \%$ reporting unprotected exposure with Covid positive patient. $70 \%$ were not sure about unprotected exposure and $52.5 \%$ were unsure about acquiring infection at work-place or in community. Almost 57.5\% reported positive on several psychological stress parameters. About $42.5 \%$ had no psychological counselling after testing positive.

Table 1 Age Distribution of COVID19 infected health care professionals

\begin{tabular}{llll}
\hline Age-group & $20-30$ years & $30-40$ years & Total \\
\hline No & 36 & 4 & 40 \\
\hline
\end{tabular}

Table 2 Gender-wise distribution of COVID19 infected health care professionals

\begin{tabular}{llll}
\hline Gender & Male & Female & Total \\
\hline Number & 16 & 24 & 40 \\
\hline
\end{tabular}


Table 3 Role of COVID19 infected health care professionals

\begin{tabular}{lllllllll}
\hline $\begin{array}{l}\text { HCP } \\
\text { role }\end{array}$ & $\begin{array}{l}\text { Resident } \\
\text { doctor }\end{array}$ & $\begin{array}{l}\text { Emergency } \\
\text { medicine }\end{array}$ & Anesthetist & $\begin{array}{l}\text { Technician } \\
\text { ICU }\end{array}$ & $\begin{array}{l}\text { Nurse in Covid } \\
\text { ICUrse in Covid } \\
\text { ward }\end{array}$ & $\begin{array}{l}\text { Nurse in non- } \\
\text { Covid ICU }\end{array}$ & $\begin{array}{l}\text { Nurse in non-Covid } \\
\text { ward }\end{array}$ \\
\hline No & 5 & 1 & 1 & 10 & 13 & 6 & 2 & 2 \\
\hline
\end{tabular}

Quarantined asymptomatic HCPs reported anxiety, loneliness and boredom. Those who developed symptoms experienced fear, insomnia, irritability and anger. Fear of infecting relatives or room partners was a common theme. Stigmatisation or avoidance by family, friends or neighbours was reported by $72.5 \%$. About $15 \%$ reported considering leaving their jobs and around $7.5 \%$ reported having suicidal thoughts after testing positive. In the suggestions section in questionnaire, infected HCPs advised others to pay attention to hand hygiene and wearing PPEs and maintain social distancing with colleagues at work and avoid eating together.

\section{Discussion}

Severe Acute Respiratory Syndrome Coronavirus 2 (SARS-CoV-2) can attack lung cells through receptor entries mainly by binding of its spike (S) protein with angiotensin converting enzyme-2 (ACE2) [3]. Human to human mode of transmission is by droplets, respiratory secretions, and direct contact with cases or fomites [5]. Presence of this virus in host cells may initiate various protective responses leading to pneumonia and Acute Respiratory Distress Syndrome [5]. As of June 13, 2020, we have approximately 7,553,182 confirmed cases worldwide with deaths numbering up to $4,23,349$. The situation in India is no exception with $3,08,993$ confirmed cases and around 8884 total deaths [9]. The incubation period of this disease ranges from 1 to 12.5 days (with median estimates of 5 to 6 days), but can be as long as 14 days [6]. The affirmed mortality rate is around $3.4 \%$ as of March 2020 . There is no room for complacency if one considers the colossal loss to human life caused by 1918 influenza pandemic with a case-fatality ratio of less than 5\% [10]. No sooner than the knowledge of the disease was acquired the WHO and national health bodies everywhere promulgated the concept of social distancing and the practice of hygiene and physical protection, with use of alcohol-based sanitizers, handwash, use of mask, gloves and personal protective equipment (PPE).

Clinical spectrum of COVID has been observed to range from asymptomatic to symptomatic with febrile myalgia to ARDS at far end of spectrum. Most commonly encountered symptoms include fever, fatigue and dry cough. Other clinical manifestations include anorexia, dyspnoea, cough, myalgias, sore throat, rhinorrhoea, headaches and less frequently nausea and diarrhoea, seen in mild to moderate forms of the disease. Patients with dysgeusia, hyposmia or even isolated anosmia with complete absence of other symptoms have also been reported [11, 12]. As these are fairly common presentations to otolaryngologists, they are at high risk of contracting infection while examining patients.

In this study, the first HCP who tested positive was an otolaryngology resident. Hospital infection control team's response was fast and all otolaryngology faculty and residents who were in contact, including her room partner were asked to self-isolate and underwent testing. ENT OPD services were closed for 5 days and contact tracing was done meticulously. It was not clear whether she acquired infection at work-place or out of it. Her room partner and co-resident had assisted in tracheostomy in a cardiac patient who later on tested Covid19 positive. But the room partner herself had tested negative.

Otolaryngologists may get infected in outdoor clinic or in ICU, attending to emergencies or in OR while doing surgeries. The virus has been shown to be viable in aerosols for $3 \mathrm{~h}$ and to be more stable on steel and plastics then on copper and cardboard remaining viable up to $72 \mathrm{~h}$ on steel surfaces [13]. If asymptomatic, otolaryngologists can infect several others at work. Viral transmission via the use of instruments, even the ones commonly used in OPDs such as tongue depressors, stethoscopes and sphygmomanometers is a high possibility. As such, otolaryngologists are advised to exercise utmost care and refrain from examining nose, throat and avoid aerosol generating procedures (AGPs), where possible. Even potentially vital procedures like tracheostomies have been asked to be deferred or avoided where deemed unnecessary by most national guidelines of otolaryngologist's association. The importance of proper training regarding protective and operative equipment, fitting of masks, donning and doffing of PPEs cannot be understated. Other recommendations include meticulously planned surgery wherever possible in negative pressure ICUs, negative pressure ORs or OT complexes with dedicated patient transport routes, reduced time of exposure to intra or postoperative aerosolization, a dedicated and well experienced team and appropriate measures of postoperative waste disposal and decontamination [14].

The risks of acquiring COVID19, for anaesthetists and those involved in emergency management is high as they 
have to undertake AGPs like intubation and administration of oxygen by masks. Despite following all precautions an anaesthetist and emergency medicine team physician were infected. Yet, exposure through aerosolization of virus in OR and emergency settings can occur. If asymptomatic or in pre-symptomatic phase, infected HCPs in emergency can potentially spread infection to several critical patients. Also if they have to be quarantined along with their contacts it may cause severe strain on emergency team.

Since anesthetists and intensivists are playing a pivotal role in helping fight this war, reduction in the time of exposure should be practiced at every cost without compromising the protective measures. Avoidance of general anaesthesia wherever possible by avoiding electives, can greatly help. Decreased intubation time, reducing the delay in intubation and curtailing the number of multiple attempts can be achieved if these procedures are handled by experts with adequate use of neuromuscular relaxants and observance of rapid sequence anesthesia.

The danger of residents in general medicine or paediatrics passing on infection to patients once positive is real and hence utmost precaution is needed in sanitising hands and all appliances used to examine patients.

In the study 23 members of nursing staff and 10 radiology technicians were detected positive for COVID19. Most of them felt that they had acquired infection at workplace while some were unsure. Importance of nursing and paramedical staff working as 'super-spreader' cannot be understated. Nurses and attendants not only look after patients but also handle equipment, instruments, drugs and other material including waste, in OPDs, wards and ICUs alike. This only leads to exponential rise in number of people infected through even a single HCP. A news-report of 49-year-old doctor testing positive after exposure to a COVID positive female and eventually leading to isolation of around 1100 people, including HCPs, for 14 days in the national capital, Delhi had recently emerged [15]. This further emphasizes importance of containment and isolation of infected HCP.

The situation has been accelerating globally with a possible doubling rate of every 3 to 4 days. The numbers of HCP exposed to the disease has also risen, with a consequent surge in numbers of HCP quarantined and a resultant decrease in strength of the doctors and the team handling the patients on the frontline. As reported by CDC, by April 16, 2020, 9,282 U.S. doctors, nurses and other health care professionals had contracted the coronavirus, and 27 had died. After analysing data on their exposure to Covid19 cases, $780(55 \%)$ were found to have been exposed only in health care settings [16]. In Italy, $20 \%$ of responding HCP were infected with some succumbing to the disease. According to a WHO situation report on April 82020 , 22,073 HCPs across 52 countries have been infected by
COVID [17]. In a world of established scarcity of medical professionals, decrement in number of doctors and nurses, puts immense moral stress on healthier counterparts.

Discussion with medical staff have highlighted physical and mental exhaustion, torture of difficult triage decisions accompanied with not only pain of losing patients but also colleagues with constant fear of acquiring the infection [18]. Although most HCPs evidently acquired infection through work-place exposures, household and community transmission cannot be denied with increase in numbers everywhere.

In this study $57.5 \%$ HCPs revealed psychological stress subsequent to testing positive and being isolated. Mental health issues have always been sequelae to large scale disasters such as pandemics. SARS patients had disturbed psychological health immediately post being infected or shortly after hospital discharge [19]. Increase in period of confinement is associated with worse psychological health outcomes [20]. Escalation in lockdown periods causes increase in depression, posttraumatic stress disorder (PTSD), substance use disorder, a broad range of other mental and behavioral disorders, domestic violence or even child abuse [21, 22].

Association of social stigma results in resistance from patients and their family in sharing their illness status and seeking health care early. In this study too HCPs admitted to perception of stigma from friends and relatives. There have been instances of HCPs working in COVID facilities, being denied entry to their residential premises in Indian cities. This can further aggravate stress encountered by healthcare workers. The role of social media in both uplifting and distressing people in complete isolation and those in-home quarantine cannot be over emphasized. Uplifting posts showing HCPs dancing or enjoying their work will definitely motivate other HCPs, Distressing forwards of HCPs suffering should be avoided at all costs. Ensuring availability of PPEs and creating safe atmosphere for HCPs is the responsibility of every institution and nation.

The role of prevention cannot be over emphasized especially nosocomial spread through HCPs. The WHO and governments world over are constantly working for development of vaccines. The utility of drugs like hydroxychloroquine and anti-virals is still questionable. As such, strict adherence to barrier precautions and social distancing remains our best bet.

\section{Conclusion}

Healthcare workers being at frontline of exposure to corona patients are at increased risk of developing COVID19 infections. When infected, they need to be taken out of 
work force to prevent infection to colleagues and patients, which puts a severe strain on their teams. Attention to their workplace safety and psychological well-being is the need of the hour.

\section{Clinical Significance and Recommendations}

Infection with COVID19 and mortality among HCPs pose a great threat in the pandemic and may lead to complete breakdown of health care as well as increase in HCPs seeking care for mental stress. Finding of asymptomatic cases, especially among HCP is not only a challenge but any delay in identification, can lead to prolongation of the chain of transmission with more HCPs getting infected.

HCPs are working under tremendous stress in this pandemic and it is necessary to combat fear with facts and work towards safe WORK atmosphere so that they can discharge their duties to best of their ability. The importance of psychological wellbeing of the national healthcare workforce cannot be neglected in this warlike situation. Efforts like 'Minding our minds during COVID 19' taken by the MOHFW should be brought to light. Measures should be instituted to help curb the physical stress and anxiety impending on the HCP and general public related to COVID. HCPs have a right to safety at workplace and all efforts should be made at operational and administrative levels to minimize the chance of getting infected. As high viral load in COVID 19 infection is associated with more serious infections and deaths in HCWs all elective surgeries with risk of aerosolization are best deferred. Also, there is need for reducing workload and where possible assign duties by rotation in staggered manner. While high risk procedures are best done by senior, more experienced staff, the very senior and those with co-morbidities are best assigned low-risk work in patient care.

The importance of social distancing, maintenance of physical barrier, apt knowledge of the disease, use of proper PPEs, proffering 'Telemedicine' wherever possible and care of mental health in these afflicted times cannot be underestimated.

\footnotetext{
Author contributions Study idea and design: YGD, BAS; Data acquisition: YGD, BAS, and SD; Data analysis: YGD; Manuscript: YGD, BAS; Critical review of the manuscript: YGD, TYD; Final approval: All authors.
}

Funding No funding was obtained for the conduct of the study.

\section{Compliance with Ethical Standards}

Conflict of interest The authors declare that there were no conflicts of interests whatsoever in the conduct or writing of this research.
Ethics Approval Institutional Ethical Clearance was obtained for the study.

Consent to Participate Informed consent was obtained from all participants.

\section{References}

1. Yuen KS, Ye ZW, Fung SY, Chan CP, Jin DY (2020) SARSCoV-2 and COVID-19: the most important research questions. Cell Biosci 10:40. https://doi.org/10.1186/s13578-020-00404-4

2. Moorthy V, Henao Restrepo AM, Preziosi MP, Swaminathan S (2020) Data sharing for novel coronavirus (COVID-19). Bull World Health Organ 98(3):150. https://doi.org/10.2471/BLT. 20.251561

3. Astuti I, Ysrafil. (2020) Severe Acute Respiratory Syndrome Coronavirus 2 (SARS-CoV-2): an overview of viral structure and host response. Diabetes Metab Syndr 14(4):407-412. https://doi.org/10.1016/j.dsx.2020.04.020

4. Lu R, Zhao X, Li J, Niu P, Yang B, Wu H et al (2020) Genomic characterisation and epidemiology of 2019 novel coronavirus: implications for virus origins and receptor binding. Lancet 395(10224):565-574. https://doi.org/10.1016/S0140-6736(20)30251-8

5. Guo YR, Cao QD, Hong ZS, Tan YY, Chen SD, Jin HJ, Tan KS, Wang DY, Yan Y (2020) The origin, transmission and clinical therapies on coronavirus disease 2019 (COVID-19) outbreak-an update on the status. Mil Med Res 7(1):11. https://doi.org/10.1186/s40779-020-00240-0

6. Li Q, Guan X, Wu P, Wang X, Zhou L, Tong Y et al (2020) Early transmission dynamics in Wuhan, China, of novel coronavirusinfected pneumonia. N Engl J Med 382(13):1199-1207. https://doi.org/10.1056/NEJMoa2001316

7. Wang C, Horby PW, Hayden FG, Gao GF (2020) A novel coronavirus outbreak of global health concern. Lancet 395(10223):470-473

8. Zhong NS, Zheng BJ, Li YM, Poon LL, Xie ZH et al (2003) Epidemiology and cause of severe acute respiratory syndrome (SARS) in Guangdong, People's Republic of China, in February, 2003. Lancet 362(9393):1353-1358

9. World Health Organization. Coronavirus disease 2019 (COVID19) Situation report-145. https://www.who.int/docs/ default-source/coronaviruse/situation-reports/20200613-covid-19sitrep-145.pdf?sfvrsn=bb7c1dc9_2. Accessed 13 June 2020

10. Viboud C, Eisenstein J, Reid $\mathrm{AH}$, Janczewski TA, Morens DM, Taubenberger JK (2013) Age- and sex-specific mortality associated with the 1918-1919 influenza pandemic in Kentucky. J Infect Dis 207(5):721-729. https://doi.org/10.1093/infdis/jis745

11. Liu K, Fang YY, Deng Y, Liu W et al (2020) Clinical characteristics of novel coronavirus cases in tertiary hospitals in Hubei Province. Chin Med J. https://doi.org/10.1097/CM9.0000000 000000744

12. $\mathrm{Xu} X W, \mathrm{Wu} X X$, Jiang $\mathrm{XG}, \mathrm{Xu} \mathrm{KJ}$ et al (2020) Clinical findings in a group of patients infected with the 2019 novel coronavirus (SARS-Cov-2) outside of Wuhan, China: retrospective case series. BMJ. https://doi.org/10.1136/bmj.m606

13. van Doremalen N, Bushmaker T, Morris DH, Holbrook MG et al (2020) Aerosol and surface stability of SARS-CoV-2 as compared with SARS-CoV-1. N Engl J Med 382(16):1564-1567

14. Du Z, Wang T (2020) Infection prevention and control in perioperative patients during the COVID-19 pandemic: protocol from a Tertiary General Hospital. J Minim Invasive Gynecol S1553-4650(20):30191-30196. https://doi.org/10.1016/j.jmig. 2020.04 .016 
15. Staff Reporter. (2020) Coronavirus: Mohalla clinic doc tests +ve; 1100 people in Delhi asked to home quarantine. The Hindu, March 26. https://www.thehindu.com/news/cities/Delhi/corona virus-mohalla-clinic-doc-tests-ve-1100-people-in-delhi-asked-tohome-quarantine/article31171612.ece.

16. CDC COVID-19 Response Team (2020) Characteristics of health care personnel with COVID-19-United States, February 12-April 9, 2020. MMWR Morb Mortal Wkly Rep 69(15):477-481. https://doi.org/10.15585/mmwr.mm6915e6

17. World Health Organization. (2020) Coronavirus disease 2019 (COVID19) Situation report-82. https://www.who.int/docs/ default-source/coronaviruse/situation-reports/20200411-sitrep82-covid-19.pdf?sfvrsn=74a5d15_2. Accessed 11 Apr 2020.

18. Yin Q, Sun Z, Liu T et al (2020) (2019) Posttraumatic stress symptoms of health care workers during the corona virus disease. Clin Psychol Psychother 27:384-395. https://doi.org/10.1002/ cpp. 2477
19. Chua SE, Cheung V, McAlonan GM, Cheung C et al (2004) Stress and psychological impact on SARS patients during the outbreak. Can J Psychiatry 49(6):385-390

20. Brooks SK, Webster RK, Smith LE, Woodland L et al (2020) The psychological impact of quarantine and how to reduce it: rapid review of the evidence. Lancet 395(10227):912-920

21. Tracy M, Norris FH, Galea S (2011) Differences in the determinants of posttraumatic stress disorder and depression after a mass traumatic event. Depress Anxiety 28(8):666-675

22. Galea S, Ahern J, Resnick H (2002) Psychological sequelae of the September 11 terrorist attacks in New York City. N Engl J Med 346(13):982-987

Publisher's Note Springer Nature remains neutral with regard to jurisdictional claims in published maps and institutional affiliations. 